\title{
Wissenschaftliche Beiträge
}

\section{Herausforderung Rechtsgeschichte}

\author{
Susanne Hähnchen *
}

Zusammenfassung: Man kann heute in Deutschland Juristin oder Jurist mit Bestnoten werden, ohne sich jemals mit Rechtsgeschichte beschäftigt zu haben. Grundlagenfächer im Allgemeinen werden zwar theoretisch als wichtig angesehen. Im deutschen universitären Studium spielen sie jedoch eine untergeordnete und in den Klausuren der staatlichen Examina keine Rolle. Nur in der mündlichen Prüfung besteht ein relativ geringes Risiko, dass z.B. rechtshistorische Fragen gestellt werden. Studierende lernen aber grundsätzlich nur das, was auch tatsächlich geprüft wird. Dies ist angesichts der Stofffülle durchaus nachvollziehbar. Lehrende der Rechtsgeschichte können daher nicht einfach auf die Examensrelevanz ihres Themas verweisen. Sie müssen alle didaktischen und persönlichen Möglichkeiten ausschöpfen, um von ihrem Fach zu überzeugen.

\section{A. Einführung}

Im Folgenden wird eine rechtsdidaktische Innenperspektive der Rechtsgeschichte eingenommen bzw. die Herausarbeitung von Gemeinsamkeiten und Besonderheiten zur allgemeinen Rechtsdidaktik versucht (unter C.). Notwendig ist jedoch zunächst die Betrachtung aus der Außenperspektive (unter B). Denn die Stellung der Rechtsgeschichte in der juristischen Ausbildung hat große Bedeutung für das Lehren und Lernen der Inhalte. Es wird dabei auf eine Diskussion der juristischen Ausbildung bzw. ihrer Reform verzichtet ${ }^{1}$ und nur skizziert, welchen Einfluss die allgemeinen Rahmenbedingungen haben.

Auf die Bedeutung von Praxisorientierung in der universitären Lehre und ihr Verhältnis zu den Grundlagen soll hier nicht näher eingegangen werden. ${ }^{2}$ Man kann und sollte zwar reale Rechtsfälle aus der Geschichte zu einem der Gegenstände des Unterrichts bzw. der Reflexion machen. ${ }^{3}$ Die Forderung nach mehr Praxisbezug meint jedoch etwas anderes, nämlich Handlungswissen im geltenden Recht. Fest-

* Susanne Hähnchen ist Professorin an der Fakultät für Rechtswissenschaften der Universität Bielefeld mit Interesse an Forschung und Lehre für Zivilrecht, Grundlagen (Rechtsgeschichte, Rechtstheorie) sowie praxisorientierte Juristenausbildung. 2018 zusammen mit Thomas Riehm (Passau) Ars-legendi-Fakultätenpreis Rechtswissenschaft. Ich danke Herrn stud. iur. Marco Birkholz für seine Unterstützung.

1 Schon Gierke (ab 1911 v. Gierke) schrieb zur damaligen Diskussion der juristischen Studienordnung in: Schmollers Jahrbuch 1877, S. 1: „Wesentliches Neues kann nicht gesagt werden. “, fuhr aber fort: „Dennoch, so undankbar die Aufgabe ist, es ist Pflicht, in dem beschwerlichen Kampf nicht zu ermüden. Immer wieder muß das, was Noth thut, vor die Augen gestellt werden.“

2 Dazu Hähnchen, in: Anwaltsblatt Karriere 2017, S. 64; dies., in: GJLE 2017/2018, S. 1 ff.; dies., in: NJW-aktuell 4/2019, S. 19.

3 Beispiele, die ausgehend vom einzelnen Rechtsfall grundlegende Fragen behandeln, in: Luminatil Falk/Schmoeckel (Hrsg.). 
zuhalten ist jedenfalls, dass Grundlagen und Praxisorientierung keinen Gegensatz darstellen, sondern zwei sich ergänzende Aspekte guter Ausbildung. ${ }^{4}$

\section{B. Probleme}

\section{Zweiteilung: Universitäres Studium - staatliches Examen}

Rechtsgeschichte und andere Grundlagenfächer leiden in besonderer Weise unter der Aufspaltung der juristischen Ausbildung, d.h. darunter, dass universitäres Studium und abschließende Prüfung weitestgehend geteilt sind. In den Ausbildungsund Prüfungsordnungen zählen die „rechtswissenschaftlichen Methoden und (...) philosophischen, geschichtlichen und gesellschaftlichen Grundlagen "5 zu den Pflichtfächern und damit zum Prüfungsstoff. Das klingt zwar gut, hilft aber wenig. Denn die Bedeutung eines juristischen Teilgebietes im Studium wird vom Ende her gedacht. Entscheidend ist, ob ein Thema auch „examensrelevant“ ist. So verführt beispielsweise die Tatsache, dass nur eine von sechs Examensklausuren das Strafrecht $^{6}$ zum Gegenstand hat, manche zivilrechtlichen Kollegen und Kolleginnen, damit den Studierenden die Wichtigkeit ihres eigenen Fachs zu begründen. Schließlich gibt es mehr zivilrechtliche Examensklausuren.

Während des Studiums müssen in der Regel nur eine oder zwei Semesterabschlussklausuren bestanden werden und damit sind gleich alle Grundlagen (Rechtsgeschichte, Rechtssoziologie, Rechtsphilosophie, Ökonomische Analyse des Rechts u.a. ${ }^{7}$ ) erledigt. Man kann daher in das Examen gehen, ohne sich jemals mit Rechtsgeschichte zu beschäftigen.

Diese Grundlagen-Scheine werden zudem typischerweise am Anfang des Studiums „abgehakt“, was suboptimal ist. Denn den Lernenden fehlen noch elementare juristische Kenntnisse, wodurch eine kritische Reflexion erheblich erschwert wird. Außerdem braucht es längere Zeit, Neugier und Geduld, um sich auf dem sonstigen Studium so ferne Themen wie die Antike, das Mittelalter oder selbst das

4 Wissenschaftsrat, Perspektiven der Rechtswissenschaft in Deutschland, 2012, S. 7 f., $32 \mathrm{ff} ., 53 \mathrm{f}$., 60 ff., der eine Stärkung von beidem, aber zu Recht auch mehr Interdisziplinarität und eine internationalere Ausrichtung fordert. Schiemann, in: FS Haase, S. 193 (198, 200 f.) betont, dass gerade eine anwaltsorientierte Juristenausbildung die Rechtsgeschichte benötige. Kritisch Funke in: NJW 2018, S. 1930 (1931): Mehr Praxis gehe zu Lasten der Theorie. Gegen diese Behauptung sprechen Erfahrungen, vgl. Hähnchen, in: GJLE 2016, S. 81 (82); tatsächlich berichten die Studierenden, wie sehr ihnen die Teilnahme an der Studentischen Rechtsberatung Bielefeld für ihr Studium im Allgemeinen hilft. Für eine grundsätzliche Trennung - Wissenschaft (nur) an den Universitäten, „praxisnahe Juristenausbildung “ an die Fachhochschulen - hingegen Stolleis, in: JZ 2013, S. 712 (714) und Oestmann, in: FAZ v. 3.12.2014, www.faz.net/-gyq-7wq9 b. Dagegen zu Recht Lege, in: NJW-aktuell 14/2017, S. 19: „Wissenschaft findet vielmehr überall statt, wo man reflektiert über Probleme nachdenkt".

5 \5 a Abs. 2 S. 3 DRiG. Ähnlich die Justizausbildungsgesetze der Länder, z.B. $\$ 11$ Abs. 3 JAG NRW.

6 Zum Problem des Strafrechts vgl. Hefendehl, in: ZDRW 2017, S. 245 (247).

7 Genauer zum „Kanon der Grundlagenfächer“ Lepsius, in: ZDRW 2016, S. 206 (208 ff.). 
20. Jahrhundert einzulassen. Diese Zeit fehlt anscheinend den meisten Studierenden. ${ }^{8}$

In der ein bis zwei Jahre dauernden - und damit einen erheblichen Teil des Studiums einnehmenden - Examensvorbereitung haben Grundlagenfächer erst recht schlechte Karten, eben da sie in den staatlichen Klausuren nicht abgeprüft werden. Einen Gedanken an Rechtsgeschichte verlieren Examenskandidaten meist nur, wenn zu ihrem Erschrecken ein Rechtshistoriker oder eine Rechtshistorikerin in der Kommission für die mündliche Prüfung sitzt. ${ }^{9}$ Dann ist es jedoch zum Lernen zu spät.

An fast der Hälfte der juristischen Fakultäten gibt es keinen Grundlagen-Schwerpunktbereich, ${ }^{10}$ der aktuell noch $30 \%$ der Examensnote ausmacht. Selbst für an den Grundlagenfächern Interessierte fehlt dann in der letzten Phase des Studiums meist jegliches Angebot an Grundlagenfächern.

\section{Verlorene Bedeutung}

Im 19. Jahrhundert hatte die deutsche Rechtswissenschaft international einen hervorragenden Ruf und die entstehenden Kodifikationen, insbesondere des Handelsund später des Bürgerlichen Rechts, galten als vorbildlich. Die bedeutendsten deutschen Juristen wie Friedrich Carl v. Savigny, Bernhard Windscheid, Rudolf v. Jhering und Otto $v$. Gierke waren das, was man heute als Rechtshistoriker bezeichnen würde. Allerdings gab es keine scharfe Trennung zwischen Vergangenheit und Gegenwart. Lange dominierte das römische Recht die Rechtspraxis. Daneben erstarkte - nicht zuletzt aufgrund des national-politischen Hintergrundes im territorial zersplitterten Deutschland - die deutsche Rechtsgeschichte. Die Wichtigkeit historischer Kenntnisse spiegelte sich in der Besetzung der Lehrstühle überwiegend mit Rechtshistorikern wieder. ${ }^{11}$

Seit den großen Kodifikationen, insbesondere seit dem Inkrafttreten des BGB zum 1.1.1900, nahm jedoch die Bedeutung rechtshistorischer Kenntnisse in der Praxis und im akademischen Unterricht kontinuierlich ab. ${ }^{12}$ Dementsprechend verschob

8 Stolleis, in: JZ 2013, S. 712: „und, nebenbei, oft auch die intellektuelle Neugier, sich auf Fragen jenseits des „Examensrelevanten“ einzulassen.“ Ähnlich schon 1877 - als es noch keine Massenuniversität gab - Gierke, in: Schmollers Jahrbuch 1877, S. 2 über die Studierenden seiner Zeit: „halten sie es für einen Verrath an Stand und Jugend, in Vorlesungen oder durch Bücher mit der Wissenschaft in intimere Berührung zu treten ... die berüchtigten Maschinerien des geistlosen Erlernens"; auch wenn er (S. 3) lobt, dass („primitive Anfänge einer Besserung“) die Zahl derjenigen gestiegen sei, die an Disziplinen teilnehmen, welche im Examen keine oder eine geringe Rolle spielen. Man sollte die Ewigkeit dieser Beschwerden vielleicht hinterfragen, d.h. wer sich warum über wen beschwert.

9 Lesenswert dazu der kurzweilige Beitrag von Oestmann, in: Ad Legendum 2019, S. 258 ff.

10 Ausführlich zu den vorhandenen, sehr unterschiedlichen Modellen Lepsius, in: ZDRW 2016, S. 206 (221 ff.).

11 Genauer dazu Lepsius, in: ZDRW 2016, S. 206 (S. 207 mit Fn. 5). Vgl. auch Stolleis, in: JZ 2013, S. 712 (713).

12 Ausführlicher dazu Schiemann, in: FS Haase, S. 195 ff. Bereits 1877 warnte Gierke in: Schmollers Jahrbuch 1877, S. $31 \mathrm{f}$. vor dem „handwerksmäßigen Niedergang“ und der wissenschaftlichen Verflachung durch die von ihm auch gewünschten Kodifikationen zur Herstellung der Reichseinheit. 
sich der Schwerpunkt der universitären Lehre, weg von historischen Quellen - hin zum positiven Recht, was ein großes Problem für die Vertreter der Rechtsgeschichte wurde. ${ }^{13}$ Dies spiegelt sich auch in der seit den 1990er Jahren nochmals deutlich gesunkenen Zahl von rechtshistorischen Professuren wieder. ${ }^{14}$

Aber bedeutet die geringe Präsenz auch eine fehlende Relevanz eines Faches?

\section{Wozu dann noch Rechtsgeschichte?}

Juristische Bildung ${ }^{15}$ und ein grundlegendes Verständnis der Veränderbarkeit des Rechts sowie seiner sozialen, kulturellen, wirtschaftlichen und politischen Bedingtheit sollten es eigentlich nicht nötig haben, sich erklären zu müssen. Auf die zahlreichen Publikationen zur Verteidigung der Rechtsgeschichte soll daher hier nur knapp verwiesen werden. ${ }^{16}$ Auch der Wissenschaftsrat hat 2012 die Bedeutung der Grundlagenfächer betont und ihre Stärkung in der Ausbildung gefordert. ${ }^{17} \mathrm{Neu}$ ist die Kritik an einer auf gerade geltende Gesetze beschränkten Rechtswissenschaft übrigens nicht: Berühmt sind etwa die bereits im 19. Jahrhundert gehaltenen Vorträge dazu von Kirchmann ${ }^{18}$ und Jhering ${ }^{19}$.

Den Rechtfertigungszwang teilt sich die Rechtsgeschichte mit dem Geschichtsunterricht. ${ }^{20}$ Offenbar ist es bereits an den weiterführenden Schulen schwierig, junge Menschen für Geschichte zu interessieren und zu begeistern. Das macht es den an Hochschulen Lehrenden nicht gerade leichter. Jedenfalls muss man den Studierenden immer wieder erklären, warum sie etwas lernen und verstehen sollten, das gar nicht mehr gilt und ohnehin höchstens gelegentlich in der mündlichen Prüfung vorkommt. Anderenfalls hat man sie sehr schnell verloren.

\section{Vorurteile und fehlende Vorkenntnisse}

Passend zum Effizienzgedanken heutiger Hochschulpolitik hält sich unter Studierenden das Gerücht, dass sie ihren künftigen Arbeitgebern erklären müssten, warum sie sich mit „so was“ beschäftigt und nicht im geltenden Recht spezialisiert hätten, wenn sie ihre Zeit vertiefter für Rechtsgeschichte verwenden. In Evaluationen zu den großen Veranstaltungen zu Beginn des Studiums kann man nachlesen,

13 Hähnchen, Rechtsgeschichte, Rn. 1-3, 739.

14 Lepsius, in: ZDRW 2016, S. 206 (207 ff.). Gutmann, in: JZ 2013, S. 697 spricht von einem „rollback" seit den 1980er Jahren.

15 So auch die Formulierung des Wissenschaftsrats, Perspektiven der Rechtswissenschaft in Deutschland, S. 57.

16 Statt Vieler genannt seien die Beiträge in Eckert (Hrsg.), Der praktische Nutzen der Rechtsgeschichte, FS Hattenhauer, sowie in der www.zeitschrift-rechtsgeschichte.de; jüngst etwa Baldus, in: JZ 2019, S. $633 \mathrm{ff}$.

17 Wissenschaftsrat, Perspektiven der Rechtswissenschaft in Deutschland, S. $58 \mathrm{ff}$.

$18 v$. Kirchmann, Die Werthlosigkeit der Jurisprudenz als Wissenschaft, 1848, S. 23: „Drei berichtigende Worte des Gesetzgebers, und ganze Bibliotheken werden zu Makulatur.“

$19 v$. Jhering, Ist die Jurisprudenz eine Wissenschaft?, 1868.

20 Vgl. die Keynotes in Sandkühler/Bühl-Gramer et al. (Hrsg.), S. 43 ff. 
dass mehrheitlich nur der Schein zur Veranstaltung motiviert hat ${ }^{21}$ - was ja vielleicht auch in manchen dogmatischen ${ }^{22}$ Fächern nicht so viel anders ist. Wer weiß schon immer, was auf ihn/sie zukommt bzw. liebt alles gleichermaßen? Aber wenn man nachfragt, dann erfährt man explizit, dass sich die meisten auch für Geschichte im Allgemeinen nicht interessieren. ${ }^{23}$ Angeblich haben sie zu viel (nur) über den Nationalsozialismus in der Schule gehört. Versucht man, daran anzuknüpfen, so zeigen sich jedoch auch für diese Zeit ähnliche Wissenslücken wie für Früheres. Es mangelt den Studienanfängern aber nicht nur an allgemeinen historischen Vorkenntnissen, sondern oft auch an einer positiven Einstellung gegenüber der Geschichte. ${ }^{24}$ Auch diese Klage ist nicht neu oder spezifisch. ${ }^{25}$

Eine längere Zeit der Beschäftigung mit rechtshistorischen Themen sowie Kenntnisse des geltenden Rechts (B.I.) können hilfreich für das Verständnis und damit für die Akzeptanz der Rechtsgeschichte sein. Das zeigt sich in Beobachtungen, die man gelegentlich wissenschaftlich daraufhin untersuchen sollte, ob sie zufällig sind. Jedenfalls äußern gerade mitten im Berufsleben stehende Praktiker oft große Begeisterung für die Rechtsgeschichte. ${ }^{26}$ Beispielsweise erhielt ich meine erste Anstellung nach dem ersten Staatsexamen in einer gutsituierten Anwaltskanzlei auch deshalb, weil es den mich befragenden Partner beeindruckte, dass ich zu einem römisch-rechtlichen Thema promovieren wollte. Auch seither treffe ich unter Praktikern und Praktikerinnen überwiegend auf Offenheit und Interesse oder gar Rechtshistoriker im Nebenberuf. Und Studierende, denen man die Rechtsgeschichte einmal „schmackhaft" gemacht hat, lassen sich später in dogmatischen Veranstaltungen und rechtshistorischen Seminaren eher darauf ein. Mit Geduld auf allen Seiten können Vorurteile und fehlende Vorkenntnisse durchaus überwunden werden. $\mathrm{Zu}$ nächst erschweren sie jedoch den Zugang zur Rechtsgeschichte.

21 Ein Beispiel unter C.II.1.

22 Dogmatik als Wissenschaft des geltenden Rechts.

23 Zur korrespondierenden Wahrnehmung der Lehrenden s.o. bei Fn. 20.

24 Bubrowski/Haneke, Eichmann? Nie gehört, in: FAZ Einspruch Magazin v. 4.4.2018; Hobe/DaunerLieb, in: F\&L 2018, S. 314: „Wer im Hörsaal auf geschichtliche oder wirtschaftliche Hintergründe ... hinweist, löst bei Studierenden Seufzen oder Langeweile aus.".

25 Thibaut, Gutachten und Stellungnahme der Heidelberger Juristenfakultät (1821) zu den damaligen Reformplänen des zuständigen (badischen) Ministeriums, veröffentlich von Braun, in: DRiZ 1990, S. 450 (452): „Bekämen wir die jungen Männer, gebildet durch Muster des Altertums, ernst und gründlich, und nicht, wie es nur zu oft der Fall ist, allerlei verschiedenartige Viel- und damit Nichtwisser, ...Vielfach auf den Schulen Sittenlosigkeit, und dann dazu eine über das ganze Weltall gehende Schwätzerei, welche den Akademien ... nicht den Bildsamen zuführt.“ Beklagt wird im Folgenden u.a. fehlende Kenntnis der „Quellen der Geschichte“. Analog äußert sich Podszun, in: ZDRW 2017, S. 224 (243) im Zusammenhang mit seiner Innerperspektive zum Kartellrecht zu fehlenden wirtschaftlichen Vorkenntnissen.

26 Vgl. auch das Interview mit dem promovierten Juristen und Romanautor Fitzek, in: karriereführer recht 1.2016, S. 14 (15) auf die Frage, ob er im Studium alles richtig gemacht habe: „Rückblickend eher nicht, denn so habe ich mich im Studium nicht eingehend mit den Themen wie Rechtsethik, Rechtsphilosophie oder auch Rechtsgeschichte befasst. Die Erfahrung zeigte einfach, dass man diese Inhalte für die Klausuren nicht brauchte. ... Heute sage ich: Das ist lebensrelevantes Wissen.“ 


\section{Vielfalt - Segen und Fluch}

Abschließend sei ein weiteres Problem erwähnt. Es gibt nicht die Rechtsgeschichte, sondern in Forschung und Lehre zahlreiche Untergliederungen: Nach Rechtsgebieten (Privatrechts-, Strafrechts- und Verfassungsgeschichte, aber z.B. auch Wirtschaftsrechtsgeschichte), nach Zeiten (Antike, Mittelalter, frühe Neuzeit und neueste Rechtsgeschichte) sowie nach äußerer und innerer Rechtsgeschichte. ${ }^{27}$ Rechtshistorikerinnen und Rechtshistoriker sind dementsprechend hochspezialisiert. Meist stehen heute deutsche Territorien bzw. zum heute in Deutschland geltenden Recht führende Entwicklungen im Zentrum. In den letzten Jahren wurde der Blick aber zunehmend europäischer und von Wenigen wird sogar noch weiter geschaut. ${ }^{28}$

Diese Vielfalt der Rechtsgeschichte enthält zwar unendliche Forschungsmöglichkeiten, auch interdisziplinär ${ }^{29}$, erschwert es den relativ wenigen Wissenschaftlerinnen und Wissenschaftlern jedoch, als Einheit ihr Fach zu vertreten und zu verteidigen. Und aus Sicht vieler Studierender ist diese Vielfalt angesichts der schon im geltenden Recht kaum beherrschbaren Stofffülle abschreckend.

\section{Wie kann Rechtsgeschichte verständlich gemacht werden?}

Es ist eine Herausforderung, Rechtsgeschichte zu lehren, wenn weder rechtliche noch historische Vorkenntnisse vorhanden sind und bei vielen Studierenden noch Desinteresse hinzukommt.

\section{Theoretische Grundlagen}

Wenn man sich dieser Herausforderung stellen und Studierende für die Rechtsgeschichte begeistern will, dann ist man zunächst ziemlich auf sich gestellt bzw. auf das, was einem die eigenen Lehrer und Lehrerinnen vorgelebt haben. Denn es gibt zwar viel Literatur zum „Wozu“, d.h. dem Wert der Rechtsgeschichte, ${ }^{30}$ aber erstaunlich wenig zur Didaktik, d.h. dem „Wie“.

Neben der Vermittlung von Faktenwissen geht es wie in der geschichtswissenschaftlichen Fachdidaktik ${ }^{31}$ um die Interpretation (Exegese) von (rechts-)historischen Quellen, Problemorientierung und Gegenwartsbezug. Methodenvielfalt ist dabei wie auch sonst ein großer Vorteil. Leider begrenzt die Form der großen Vor-

27 Zum Zusammenhang zwischen äußerer (eher allgemein-historischer) und innerer (Dogmengeschichte) Entwicklung vgl. Baldus, in: JZ 2019, S. 633 (636f.).

28 Von deutschen Rechtshistorikern vor allem auf die ägyptische und die altorientalischen Rechtskulturen.

29 Zur Attraktivität der Rechtsgeschichte für interdisziplinäre Kooperationen vgl. Lepsius, ZDRW 2016, S. 206.

30 Nachweise siehe Fn. 16. Vgl. auch Lepsius, in: ZDRW 2016, S. 206 (216 ff., 236) mwN.

31 Z.B. Günther-Arndt/Handro (Hrsg.), Geschichtsmethodik; Pandel, Geschichtsdidaktik; Barricelli/ Lücke (Hrsg.). Interessant zur Geschichte von Didaktik und Methode in der Geschichtsdidaktik: Deile, Didaktik der Geschichte, S. 5 ff.; dort S. 11 auch zum schwierigen Verhältnis zwischen universitärer und schulischer Geschichtsdidaktik. 
lesung die aktive Einbeziehung der Studierenden erheblich. Im Folgenden sollen die dennoch bei diesem Format bestehenden Möglichkeiten ausgeführt werden.

\section{Praktische Methoden}

Dass die äußeren Bedingungen nicht ideal sind (oben B.), birgt auch Chancen: In den Grundlagenfächern muss man Rechenschaft ablegen - den Studierenden und sich selbst. Lehrende müssen ihr Tun besonders reflektieren, um die Lernenden zu erreichen. Auch wenn wenig darüber geschrieben wird - die meisten Rechtshistorikerinnen und Rechtshistoriker machen sich schon länger Gedanken über gute Lehre und wenden Methoden an, die sich in der Lehre dogmatischer Fächer erst allmählich durchsetzen.

\section{Interesse und Motivation evaluieren}

Es mag banal klingen, dass Motivation wichtig ist. Aber gerade in den Grundlagenfächern hat man eine hohe Notwendigkeit, Interesse zu wecken und besonders wenig Zeit, die Studierenden davon zu überzeugen, dass sie genau diese Veranstaltung besuchen sollten (B.IV.). ${ }^{32}$ Selbst in den dogmatischen Fächern, die von vornherein als wichtiger gelten, ist Besucherschwund von Woche zu Woche bekannt und üblich. Wenn man jedoch eine rechtshistorische Vorlesung anbietet, dann konkurriert man zudem noch mit anderen Grundlagenveranstaltungen. Zwar schauen sich viele Studierende verschiedene Veranstaltungen an, bevor sie sich entscheiden, welche davon sie weiter belegen wollen. Aber diese Entscheidung fällt schnell, oft ausgerichtet an der Frage nach dem geringsten Lernaufwand. ${ }^{33}$ Die wenigsten Studierenden besuchen freiwillig mehr als sie müssen.

In diesem Zusammenhang sollten Lehrende nicht nur selbst erklären ${ }^{34}$, sondern auch Evaluationen nutzen. Eine Veranstaltung wird ja gerade von denjenigen Studierenden bewertet, die geblieben sind. D.h. von ihnen kann man erfahren, was sie motiviert hat.

2016 hatte ich ein neues Feedback-Instrument ausprobiert, die Bielefelder Lernzielorientierte Evaluation (BiLOE) des Zentrums für Lehren und Lernen (ZLL). Die fakultätsinternen Bögen, die man während des Semesters austeilte, hatte ich schon vorher teilweise abgewandelt, um an mehr Informationen zu kommen, wie man die Studierenden motivieren kann, aber wirklich Neues erfuhr ich nicht mehr. Daher wandte ich mich an das ZLL. Zusammen passten wir die vorhandene Evaluation an die Veranstaltung „Europäische Privatrechtsgeschichte“ an und auf meinen Wunsch wurde sie online und erst nach der Rückgabe der Semesterabschlussklausuren durchgeführt. ${ }^{35}$ Die üblichen fakultätsinternen Evaluationen sind zwar in-

32 Allgemein dazu Eickelberg, Didaktik, Rn. 80 ff., 159 ff.

33 Hufen, in: JuS 2017, S. 1 (3): Grundsatz des „geringsten Widerstands“.

34 Dazu siehe B.III. und C.II.2.

35 Zur BiLOE mit Bezug zu meiner Veranstaltung Frank, in: duz 2017, S. 73 (74). Die Abschlussklausur im WS 2015/16 hatten genau 150 Studierende mitgeschrieben; die Durchfallquote betrug 28 
zwischen auch online, finden aber nach wie vor im laufenden Semester statt. Ich halte es jedoch für wenig sinnvoll, dass die Studierenden etwas reflektieren, über dessen Erfolg bzw. Ausgang sie keine Sicherheit haben.

Auf die Frage, mit welchem Ziel die Studierenden in die Veranstaltung gegangen waren, lautete die Antwort ganz überwiegend, dass sie die Prüfung bestehen wollten. Von 51 dazu gegebenen Antworten zeugte weniger als die Hälfte von primären (14) oder zumindest teilweisen (9) inhaltlichen Interesse. Dabei sollte man berücksichtigen, dass diese Aussagen in der Rückschau erfolgten, also nachdem ich das ganze Semester den Sinn der Veranstaltung zu vermitteln versucht habe. Die ex-post-Beurteilung der Studierenden fiel allerdings sehr positiv aus.

Das primäre Ziel der BiLOE ist es, die Studierenden zur Selbstreflexion anzuregen, welche Ziele sie verfolgen und wie sie diese am besten erreichen. Deshalb ging es vor allem um Mittel und Wege, wie man erfolgreich lernt. Durch die Ausarbeitung der Fragen war ich jedoch zum ersten Mal gezwungen, in voller Klarheit zu formulieren, welche Ziele ich als Dozentin eigentlich verfolge. Dazu gleich genauer (2.). Seither beginne ich nicht nur immer damit, diese Lernziele im Hinblick auf die Privatrechtsgeschichte zu erklären, sondern ich versuche es auch in anderen Veranstaltungen.

Die Auswertung der BiLOE zur Privatrechtsgeschichte stelle ich zu Beginn jedes neuen Semesters in den digitalen Lernraum (Moodle) ein. Es motiviert die Studierenden, wenn sie sehen können, was anderen geholfen hat. Vor allem aber könnte ich den Studierenden niemals so gut erklären, dass es sinnvoll ist, die Veranstaltung zu besuchen, wie durch die Antworten ihrer Kommilitoninnen und Kommilitonen auf die Frage „Was möchten Sie zukünftigen Studierenden über diese Lehrveranstaltung gerne mitteilen?“.

\section{Lehr- und Lernziele}

Es ist in der juristischen Lehre verbreitet, einfach zu verlangen, dass die Studierenden „den Stoff“ der jeweiligen Veranstaltung bzw. den Examensstoff beherrschen sollen. Abgesehen davon, dass das wegen der Fülle in allen Rechtsgebieten nur Gedächtniskünstlern ${ }^{36}$ gegeben ist - sollte es doch wichtiger sein, juristisch denken und arbeiten zu lernen. ${ }^{37}$ Das eigentlich Wissenschaftliche sind nicht die rechtlichen Details, sondern die Art und Weise, wie man sich mit diesen beschäftigt. Mehr kritische Reflexionskompetenz und Kritikfähigkeit wird zwar immer wieder angemahnt. ${ }^{38}$ Aber woher sollen diese kommen, wenn Studierende primär Detail-

Prozent. Nach der Rückgabe und Besprechung erhielten alle, die sich zur Klausur angemeldet hatten, den Link zur Evaluation. 76 Studierende haben die Evaluation begonnen, 49 auch beendet.

36 Interessant u.a. zum Verhältnis Gedächtnis - Verstehen, aber auch (rechts-) historisch: Hamann, in: ZDRW 2016, S. $116 \mathrm{ff}$.

37 So z.B. Zwickel, in: Griebel (Hrsg.), S. 134 (142) im Zusammenhang mit dem Nutzen der Rechtsvergleichung, welche mit der Rechtsgeschichte eng verwandt ist. Zur Unwissenschaftlichkeit in der derzeitigen Ausbildung und Prüfung vgl. Hobe/Dauner-Lieb, in: F\&L 2018, S. 314 (315 f).

Wissenschaftsrat, Perspektiven der Rechtswissenschaft in Deutschland, S. 57. 
wissen anhäufen, gedrillt ${ }^{39}$ und geprüft werden und selten jemand das „Wie“ erklärt oder das „Wozu“ diskutiert? Das einzige Lernziel scheint oft zu sein, die nächste Prüfung bzw. das Examen zu bestehen. Dabei wird der Prozess des Lernens ${ }^{40}$ mit seinem Ende, der abschließenden Prüfung, verwechselt.

Auf Lerntheorien und die Bedeutung der Kommunikation von Lehr- und Lernzielen im Allgemeinen kann hier nicht eingegangen werden. ${ }^{41}$ Es ist jedoch festzustellen, dass der internalisierte Rechtfertigungszwang der Rechtsgeschichte (B.III.) auch hier von Vorteil sein kann. Denn dadurch wird nicht nur allgemein die Bedeutung des Fachs behauptet, sondern gegenüber den Studierenden werden auch konkrete Ziele bzw. deren Sinn kommuniziert. Bei der folgenden Darstellung der meines Erachtens wichtigsten Lehr- und Lernziele in einer rechtshistorischen Veranstaltung zeigt sich deren Überschneidung mit den Gegenständen des juristischen Studiums im Ganzen.

\section{a) Inhalte und Prüfungskanon}

Rechtshistorische Forschung ist oft sehr speziell, sodass sich deren Nutzen dem Laien nicht ohne weiteres erschließt. Man sollte dabei aber nicht vergessen, dass auch im geltenden Recht die Spezialisierung weit fortgeschritten und nicht jedem alles ohne Weiteres zugänglich ist. Ohne die Detailkenntnisse, die oftmals im Laufe der Zeit vergessen oder noch nie wissenschaftlich reflektiert wurden, ist ein Erkennen oder gar ein Verständnis der großen Zusammenhänge nicht möglich. Rechtshistorische Forschung muss daher nicht unmittelbar „nützlich“ sein - Lehre schon eher. Daher bietet es sich an und ist auch üblich, die Themen jedenfalls der großen Vorlesungen so zu wählen, dass sie zum Verstehen des geltenden Rechts beitragen. Beispielsweise sind die Grundrechte einfacher zu überblicken, wenn man weiß, seit wann und warum es Menschenrechte gibt. Oder der Aufbau des BGB und die darin geregelte Materie erschließt sich leichter, wenn man die immer wiederkehrenden Probleme um vertragliche Vereinbarungen, Delikt, Eigentum, Familie und Erben historisch kennengelernt hat.

Seit einiger Zeit gibt es auch Überlegungen, ob in den Justizausbildungsgesetzen ein Prüfungskanon zu der pauschalen Formulierung, dass die „geschichtlichen Grundlagen “42 Pflicht und Prüfungsstoff seien, ergänzt werden sollte. ${ }^{43}$ Darin wür-

39 Auch Lege, in: NJW-aktuell 14/2017, S. 19 wünscht sich „weniger Drill und mehr Denkendürfen mit Hintergrundwissen“. Vgl. zum „heimlichen Lehrplan“, der „(habitualisierte) Gleichförmigkeit erzeugende(n) intuitive(n) soziale(n) Praxis“ und dem „hierarchisch organisierten, strukturierten und wenig diskursiven Wissensvermittlungsstil“ Böning/Schultz, in: Boulanger/Rosenstock et al. (Hrsg.), S. 191 (192, 195). Noch drastischer die Glosse von Braun, in: JZ 2000, S. 889. Vgl. auch Fitzek, in: karriereführer recht 1.2016, S. 14 (15): „Man sollte nicht probieren, lediglich zu funktionieren, sondern auch lernen, die Dinge zu hinterfragen. Gerade als Jurist.“

40 Lat. studium = Begeisterung, Vorliebe, wissenschaftliche Bildung.

41 Vgl. dazu Eickelberg, Didaktik, Rn. 21 ff., 118 ff.

42 S.o. bei Fn. 5.

43 Die Diskussion wird eher mündlich geführt und es gibt Befürworter und Gegner. Andeutungen finden sich bei Schmoeckel/Stolte, Vorwort S. V in: Schmoeckel/Stolte (Hrsg.); Oestmann, in: Ad Legendum 2019, S. 258 (259). 
de man die wichtigsten Themen, die beherrscht werden müssten, aufzählen. ${ }^{44}$ Das würde zwar nichts an dem Umstand ändern, dass sie nur im mündlichen Staatsexamen abgefragt werden. Außerdem könnte eine Beschränkung für die rechtshistorische Vielfalt gefährlich sein, da sie eine Begrenzbarkeit des Stoffes vorspiegelt bzw. der Rechtfertigungsdruck für nicht aufgeführte Teilgebiete steigt. Andererseits sind auch heute Forschung und Lehre nicht zwingend identisch und durch einen solchen Prüfungskanon würden die Studierenden eine größere Sicherheit hinsichtlich des zu Lernenden und das Fach von Beginn des Studiums an eine höhere Bedeutung erhalten. Es wäre dann nämlich verbindlicher in seiner Examensrelevanz.

\section{b) Umgang mit (historischen) Texten}

Sobald das Stichwort „Exegese“ in einer rechtshistorischen Veranstaltung fällt, kann man eine Schockstarre bei den Studierenden beobachten. Dabei bedeutet es nur, einen Text nach bestimmten wissenschaftlichen Kriterien auszulegen. ${ }^{45}$ Der Begriff kommt aus dem Griechischen und bedeutet übersetzt Auslegung, Erklärung, Interpretation. Ziel einer Exegese ist das Verständnis eines Textes. Man sollte dabei lernen, dass ein Text ganz unterschiedliche Inhalte haben kann, abhängig von der Zeit, dem Verfasser und dem Zusammenhang.

Bei genauerem Hinsehen handelt es sich um die typische Tätigkeit von Juristinnen und Juristen, die permanent Texte (Gesetze, Willenserklärungen und Verträge, aber auch Rechtsprechung und Literatur) auslegen. ${ }^{46}$ Leider haben sie dieses juristische „Handwerk“ selten gründlich erlernt bzw. gehen dabei eher unbewusst vor. Es ist bedauerlich, dass nicht an allen juristischen Fakultäten Veranstaltungen zur Methodenlehre angeboten werden. Gesetze, wissenschaftliche Literatur sowie Rechtsprechung zu recherchieren, auszulegen, zu beurteilen und für die eigene Arbeit zu nutzen - im Studium lernt man es meist nur am Rande.

Im rechtshistorischen Zusammenhang muss man allen Rechtstexten reflektiert begegnen, will man nicht Gefahr laufen, sie falsch, d.h. ahistorisch, zu verstehen. Was man anhand von rechtshistorischen Quellen lernen kann, hat also Bedeutung auch für den Umgang mit dem geltenden Recht.

\section{c) Reflexion}

Der rechtshistorische Blick ist unweigerlich kritisch. ${ }^{47}$ Neben dem faktenbezogenen Wissenserwerb und dem Umgang mit Texten geht es in der rechtshistorischen

44 An der Wiener Fakultät gibt es - allerdings sehr detailreiche - Listen des universitären Prüfungsstoffes für die Verfassungsgeschichte (https://backend.univie.ac.at/fileadmin/user_upload/i_rechtsgeschich te/PDF/Stichw_Verfassungsges.pdf) und für das Privatrecht (https://backend.univie.ac.at/fileadmin/us er_upload/i_rechtsgeschichte/PDF/Stichw_Privatrechtsges.pdf ). Eine Orientierung könnte z.B. auch Schmoeckel/Stolte (Hrsg.), Examinatorium Rechtsgeschichte sein, mit 20 Gesetzen, 20 Rechtsfragen, 20 Juristen, 20 Herrschaftsformen, 20 Wegen der Rechtsfindung und -durchsetzung sowie 20 Schulen und Epochen.

45 Zur Technik der rechtshistorischen Exegese genauer Hähnchen, Rechtsgeschichte, Rn. $28 \mathrm{ff}$.

46 Schon die römischen Juristen der Antike haben sich theoretisch mit der Auslegung befasst, vgl. dazu Adomeit/Hähnchen, Rechtstheorie mit Juristischer Methodenlehre, Rn. 65.

47 Genauer dazu Stolleis, in: Hof/v. Olenhusen (Hrsg.), S. 212 ff. 
Lehre vor allem darum, die Funktionen des Rechts sowie die Verantwortlichkeit von Juristinnen und Juristen zu verstehen. Dabei sollte deutlich werden, welchen Einfluss gesellschaftliche und wirtschaftliche Bedürfnisse auf die jeweiligen Regelungen haben und dass diese nicht „in Stein gemeißelt“ sind. Rechtssetzung ist politisch, Ausdruck von Machtverhältnissen und Zeitgeist, Rechtsanwendung bzw. Rechtsdurchsetzung ebenfalls. Dies zu reflektieren ist blindem Positivismus vorzuziehen und keineswegs auf die Vergangenheit beschränkt.

In diesem Zusammenhang hat das Bundesministeriums der Justiz und für Verbraucherschutz 2017 erwogen, die Zeit des Nationalsozialismus in den Vordergrund zu rücken bzw. ausdrücklich zum Pflichtstoff zu machen. ${ }^{48}$ Es spricht jedenfalls viel dafür, die Auseinandersetzung mit dem NS-Unrecht nicht auf die Rechtsgeschichte zu beschränken. ${ }^{49}$

\section{Verdeutlichung von Strukturen und Prinzipien}

Für das Verständnis von Details ist es immer hilfreich, wenn sie in Zusammenhänge eingeordnet werden. ${ }^{50}$ In der Lehre dogmatischer Fächer werden Strukturen und in verschiedenen Zusammenhängen relevante Prinzipien oft nicht ausreichend aufgezeigt, weil sie den Experten so selbstverständlich erscheinen. Auch sind die einzelnen Grundkurse (zu) wenig miteinander verknüpft. Beispielsweise die Verwandtschaft des staatlichen Strafrechts und des privaten Deliktsrechts erschließt sich für den Studienanfänger nicht ohne weiteres. Rechtsgeschichte kann und sollte auch den „großen Bogen“ erzählen, d.h. Gegenwartsbezüge herstellen und damit aufzeigen, warum es heute bestimmte Rechtsinstitute oder Problemlösungen gibt. Für das Beispiel ist es der gemeinsame Ursprung im Privatstrafrecht, welches parallel zur erstarkenden Staatlichkeit ausdifferenziert wird. ${ }^{51}$

\section{Exemplarisches Lernen}

Mindestens für die Zeit des Grundstudiums sollte Rechtslehre nicht auf Vollständigkeit angelegt sein, sondern klar Prioritäten setzen. Es ist sinnvoll, zu vereinfachen und exemplarisch zu arbeiten. Statt den Stoff in seiner Fülle auszubreiten, sollte Verständnis zentraler Problemfelder angestrebt werden. Es gibt dafür jedoch keine einheitlichen Vorgaben. Deshalb müssen die einzelnen Lehrenden selbst Mut zur Reduktion bei gleichzeitiger Gründlichkeit hinsichtlich des Behandelten haben und die Studienordnungen Strukturen zur Vertiefung bieten.

In Österreich ist die Rechtsgeschichte umfangreicher als Propädeutikum dem Studium des geltenden Rechts vorgeschaltet, was aber in Deutschland keine Chance auf Umsetzung hat und daher nicht weiter thematisiert werden soll. Aber jede

48 Vgl. dazu die Beiträge in ZDRW 1/2019 mwN; insbesondere gegen Einwände Bleckmann, in: ZDRW 2019, S. 76 (78 ff.).

49 Gusy, in: ZDRW 2019, S. 1 (11 f., 15).

50 Hufen, in: JuS 2017, S. 1 (3): „Durchblick durch Überblick“.

51 Näher dazu Hähnchen, Rechtsgeschichte, Rn. 69, 108, 557 ff., 563 ff. 
rechtshistorische Veranstaltung zwingt zu einer starken Begrenzung auf besonders relevante Themen ${ }^{52}$ - ein Aspekt, der Schule machen sollte.

\section{Visualisierung}

Ein Kennzeichen der Rechtslehre ist es, dass sie sich selten auf Gegenstände der Anschauung bezieht, sondern ganz überwiegend auf Texte. Wichtige rechtsdidaktische Mittel sind jedoch neben typographischen Gestaltungen (Strukturbilder, Tabellen und verschiedene Möglichkeiten der Hervorhebung) auch nicht-phonetische Zeichen, die nicht nur Unterhaltungs-, Aufmerksamkeits- und Erinnerungsfunktion haben, sondern die Inhalte oftmals leichter zugänglich machen, als (nur) gesprochene und geschriebene Texte..$^{53}$

Gerade in der Rechtsgeschichte stehen bildliche Darstellungen zahlreich zur Verfügung und werden von vielen Lehrenden eingesetzt. Waren dies zur Zeit meines Studiums in den 1990er Jahren noch Dias und Drucke, so bietet PowerPoint heute sogar noch einfachere Präsentationsmöglichkeiten. Dabei handelt es sich z.B. um gegenständliche Rechtsquellen ${ }^{54}$ sowie Illustrationen zu Rechtstexten und rechtlich relevanten Handlungen. ${ }^{55}$

Neben visuell wahrnehmbaren Bildern können auch interne Bilder von Bedeutung sein. ${ }^{56}$ Die Rechtsgeschichte bietet mit den Rechtssprichwörtern ${ }^{57}$ einen Schatz, den man nicht nur in rechtshistorischen Veranstaltungen einsetzen sollte. Die schriftlich überlieferten, ursprünglich für eine Mündlichkeitskultur (Oralität) stehenden Ausdrücke, Sprichwörter und Formeln waren oft verfahrensrechtlicher

52 Das gilt genauso für rechtshistorische Lehrbücher, vgl. Vorwort von Schröder zu seinem AlpmannSchmidt-Skript Rechtsgeschichte, 9. Aufl. 2013.

53 Holzer, in: Brockmann/Dietrich et al. (Hrsg.), S. 155 (156ff.) mwN. Im theoretischen und praktischen Ansatz am ausführlichsten wohl Röhl/Ulbrich, Recht anschaulich. Vgl. auch die interdisziplinären Beiträge in Hilgendorf (Hrsg.) Rechtsvisualisierung, mwN. Über 100 Jahre zurück geht die interessante Auswertung von „Visualisierungsvorstößen“" in der Rechtswissenschaft bei Ihden, in: ZDRW 2019, S. 140 (142 ff.). Zur Bedeutung von Medien und der Notwendigkeit vielfältiger Präsentationsformen in der allgemeinen Geschichtsdidaktik vgl. die Beiträge in Sandkühler/Bühl-Gramer et al. (Hrsg.), S. 413 ff.; Pandel, in: Barricelli/Lücke (Hrsg.), S. 281 ff. (286 f.).

54 Hähnchen, Rechtsgeschichte, Rn. 5. Insbesondere für die Erforschung von Rechtsordnungen, die weitgehend auf Mündlichkeit basierten, wie in früher germanischer Zeit, spielen archäologische Zeugnisse eine wichtige Rolle.

55 Berühmt sind vor allem die mittelalterlichen Bilderhandschriften. Manche gibt es (ganz oder teilweise) als Reproduktionen, z.B. Munzel-Everling (Hrsg.). Der Sachsenspiegel. Die Heidelberger Bilderhandschrift. Faksimile, Transkription, Übersetzung, Bildbeschreibung; Ebel/Fijal/Kocher, Römisches Rechtsleben im Mittelalter. Miniaturen aus den Handschriften des Corpus Iuris Civilis. Vgl. z.B. auch Schild, Folter, Pranger, Scheiterhaufen. Rechtsprechung im Mittelalter.

56 Holzer in: Brockmann/Dietrich et al. (Hrsg.), S. 155 (165 ff.) zu Konzeptmetaphern in der heutigen Alltags- und Rechtssprache.

57 Vgl. vor allem Liebs (Hrsg.) Lateinische Rechtsregeln und Rechtssprichwörter; Schmidt-Wiegand (Hrsg.), Deutsche Rechtsregeln und Rechtssprichwörter - jeweils mit Herkunft sowie Literaturhinweisen. 
Art. ${ }^{58}$ Gerade für das materielle Recht finden sich aber auch prägnante Beispiele, die wenig an ihrer heutigen Relevanz eingebüßt haben. ${ }^{59}$

\section{Verzahnung von Grundlagen und Dogmatik}

Große Vorlesungen zur Grundlegung und Vertiefung in rechtshistorischen Seminaren sind nicht alles. Eine starke Verbindung vor allem der inneren Rechtsgeschichte ${ }^{60}$ mit den „normalen“ Grundkursen - wo immer sie sich in der Lehre anbietet wäre ideal. ${ }^{61}$ Voraussetzung ist natürlich, dass der oder die Lehrende beides im Blick hat und beherrscht. Beispielsweise im öffentlichen Recht können rechtshistorische Aspekte das Verständnis von Staat, Verfassung, Grundrechten, Verwaltung usw. fördern. Im Familienrecht, das gerade in den letzten Jahrzehnten einem permanenten Wandel unterliegt, drängt sich diese Verbindung geradezu auf. Wohl nicht zufällig unterrichten auffällig viele Rechtshistoriker dieses Gebiet. Und selbst ungefragt äußern meine Studierenden in Evaluationen, dass die rechtshistorischen Bezüge für das Verständnis des geltenden Familienrechts hilfreich sind.

\section{Fazit}

Die Inhalte der Juristenausbildung sollten nicht nur nach ihrem unmittelbaren Nutzen ausgewählt werden, sondern auch eine fachspezifische Allgemeinbildung anstreben. Studium heißt mehr, als sich prüfen zu lassen. Ein rechtshistorischer Pflichtkanon ist dennoch sinnvoll.

Weil die Rechtsgeschichte ihren Platz in der juristischen Ausbildung verteidigen muss, gebraucht ihre Lehre schon jetzt viele Mittel, die auch allgemein-rechtsdidaktisch für empfehlenswert gehalten werden. Typisch juristisch ist an den geschilderten Methoden vor allem der reflektierte Umgang mit Rechtstexten als Lehr- und Lernziel. Visualisierung hat rechtshistorisch betrachtet Tradition; sie sieht sich daher weniger Vorbehalten ausgesetzt als in den dogmatischen Fächern. Wissenschaftlichkeit zeigt sich nicht darin, dass etwas schwer verständlich ist, sondern in der Arbeitsweise.

\section{Literaturverzeichnis}

Adomeit, Klaus/Hähnchen, Susanne, Rechtstheorie mit Juristischer Methodenlehre, 7. Auflage, Heidelberg 2018.

Baldus, Christian, Geschichte der Rechtsmethode - Methode der Rechtsgeschichte, in: JZ 2019, S. 633-639.

58 Hähnchen, Rechtsgeschichte, Rn. 6.

59 Ein simples Beispiel: „Einem geschenkten Gaul schaut man nicht ins Maul.“ Das Sprichwort bezieht sich auf die Praxis der Pferdehändler, beim Verkauf eines Pferdes anhand von Gebiss und Zähnen Alter und Zustand des Pferdes zu prüfen. - Noch heute ist die Haftung des Schenkers für Sachmängel wegen der Unentgeltlichkeit seiner Leistung beschränkt ( $\$ 524$ BGB).

60 Zur Unterscheidung innere-äußere Rechtsgeschichte s.o. bei Fn. 27.

61 Das fordert auch der Wissenschaftsrat, Perspektiven der Rechtswissenschaft in Deutschland S. 60 f.; ähnlich Stolleis, in: JZ 2013, S. 712 (713). 
Barricelli, Michele/Lücke, Martin (Hrsg.), Handbuch Praxis des Geschichtsunterrichts, 2 Bände, 2. Auflage, Schwalbach 2017.

Bleckmann, Frank, Das Justizunrecht des 20. Jahrhunderts gehört in die juristische Ausbildung!, in: ZDRW 1/2019, S. 76-84.

Böning, Anja/Schultz, Ulrike, Juristische Sozialisation, in: Boulanger/Rosenstock/Singelstein (Hrsg.), Studienbuch Interdisziplinäre Rechtsforschung. Eine Einführung in die geistes- und sozialwissenschaftliche Befassung mit dem Recht und seiner Praxis, S. 193-205, Wiesbaden 2019.

Braun, Johann, Ein Gutachten Thibauts zur Ausbildungsreform, in: DRiZ 1990, S. 450-452.

ders., Der Geist der modernen Juristenausbildung ist leicht zu fassen, in: JZ 2000, S. 889.

Bubrowski, Helene/Haneke, Alexander, Eichmann? Nie gehört, in: FAZ Einspruch, Magazin v. 4.4.2018, https://einspruch.faz.net/einspruch-magazin/2018-04-04/eb4b06b18dde37837b97572ac41fb8f4/?GEP $\mathrm{C}=\mathrm{s} 5$ (16.07.2019).

Deile, Lars, Didaktik der Geschichte, in: Docupedia-Zeitgeschichte, 27.01.2014, http://docupedia.de/zg/ Deile_didaktik_v 1_de_2014 (16.07.2019).

Ebel, Friedrich/Fijal, Andreas/Kocher, Gernot, Römisches Rechtsleben im Mittelalter. Miniaturen aus den Handschriften des Corpus Iuris Civilis, Heidelberg 1988.

Eckert, Jörn (Hrsg.), Der praktische Nutzen der Rechtsgeschichte. Hans Hattenhauer zum 8. September 2001, Heidelberg 2003.

Eickelberg, Jan M., Didaktik für Juristen. Wissensvermittlung - Präsentationstechnik - Rhetorik, München 2017.

Fitzek, Sebastian, Interview in: karriereführer recht 1.2016, S. 14-18.

Frank, Andrea, Ziel erreicht? Studierende reflektieren Lehren und Lernen, in: duz 05/2017, S. 73-75.

Gierke, Otto, Die juristische Studienordnung, in: Schmollers Jahrbuch 1877, S. 1-32.

Günther-Arndt, Hilke/Handro, Saskia (Hrsg.), Geschichtsmethodik. Handbuch für die Sekundarstufe I und II, 7. Auflage, Berlin 2018.

Gusy, Christoph, Aus der Geschichte lernen: NS-Unrecht im Jurastudium - Einige Vorüberlegungen und 10 Thesen, in: ZDRW 1/2019, S. 1-15.

Gutmann, Thomas, Der Holzkopf des Phädrus - Perspektiven der Grundlagenfächer, in: JZ 14/2013, S. 697-700.

Hähnchen, Susanne, Rechtsgeschichte. Von der Römischen Antike bis zur Neuzeit, 5. Auflage, Heidelberg 2016.

dies., Studentische Rechtsberatung Bielefeld - ein Werkstattbericht, in: GJLE 2016, S. 81-85.

dies., Mehr Vielfalt. Wissenschaftlichkeit und Praxisorientierung - kein Widerspruch in der Juristenausbildung, in: Anwaltsblatt Karriere 2017, S. 64.

dies., Geleitwort, in: GJLE 2017/2018, S. 1-4.

dies., Was ist gute Lehre?, in: NJW-aktuell 4/2019, 19.

Hamann, Hanjo, Gedächtniskunst in der Rechtsdidaktik. Zum Wert von Eselsbrücken für Studium, Referendariat und Rechtspraxis, in: ZDRW 2/2016, S. 116-135.

Hefendehl, Roland, Die spezifischen Herausforderungen der strafrechtlichen Lehre, in: ZDRW 4/2017, S. 245-260.

Hilgendorf, Eric (Hrsg.), Rechtsvisualisierung, Berlin 2005.

Hobe, Stephan/Dauner-Lieb, Barbara, Zukunftsfähig? Die Juristenausbildung in Deutschland, in: Forschung \& Lehre (F\&L) 4/2018, S. 314-316.

Holzer, Florian, Visualisierung im rechtswissenschaftlichen Studium, in: Brockmann/Dietrich/Pilniok (Hrsg.), Exzellente Lehre im juristischen Studium, Baden-Baden 2011, S. 155-170.

Hufen, Friedhelm, Der wissenschaftliche Anspruch des Jurastudiums, in: JuS 2017, S. 1-6. 
Ihden, Tanja, Recht auf Bilder? Visualisierung im juristischen Kontext zur Wissensvermittlung und -verarbeitung, in: ZDRW 2/2019, S. 140-164.

v. Jhering, Rudolf, Ist die Jurisprudenz eine Wissenschaft?, Wiener Antrittsvorlesung vom 16.10.1868. Aus dem Nachlass herausgegeben und mit einer Einführung, Erläuterung sowie einer wissenschaftlichen Einordnung versehen von Okko Behrens, 2. Auflage, Göttingen 2009.

Kirchmann, Julius H., Die Werthlosigkeit der Jurisprudenz als Wissenschaft (1848), online unter: http://s ammlungen.ub.uni-frankfurt.de/1848/urn/urn:nbn:de:hebis:30:2-21092 (16.07.2019)

Lege, Joachim, Vom Beruf der Juristischen Fakultäten für die Rechtswissenschaft, in: NJW-aktuell 14/2017, S. 19.

Lepsius, Susanne, Stellung und Bedeutung der Grundlagenfächer im juristischen Studium in Deutschland - unter besonderer Berücksichtigung der Rechtsgeschichte, in: ZDRW 3/2016, S. 206-241.

Liebs, Detlef (Hrsg.), Lateinische Rechtsregeln und Rechtssprichwörter, 7. Auflage, München 2007.

Luminati, Michele/Falk, Ulrich/Schmoeckel, Mathias (Hrsg.), Mit den Augen der Rechtsgeschichte: Rechtsfälle - selbstkritisch kommentiert, Zürich/Berlin 2008.

Munzel-Everling, Dietlinde (Hrsg.), Der Sachsenspiegel. Die Heidelberger Bilderhandschrift. Faksimile, Transkription, Übersetzung, Bildbeschreibung, CD-ROM, Heidelberg 2009

Oestmann, Peter, Kritik am Jurastudium. Das freie Denken kommt zu kurz, in: FAZ v. 3.12.2014, https:/ /www.faz.net/aktuell/beruf-chance/campus/jurastudium-ein-plaedoyer-fuer-mehr-selbststaendiges-den ken-13284335.html (16.07.2019).

ders., Rechtsgeschichte im mündlichen Staatsexamen, in: Ad Legendum 3/2019, S. 258-262.

Pandel, Hans-Jürgen, Geschichtsdidaktik. Eine Theorie für die Praxis, 2. Auflage, Schwalbach 2017.

Podszun, Rupprecht, Kartellrecht unterrichten, in: ZDRW 4/2017, S. 224-244.

Röhl, Klaus F./Ulbrich, Stefan, Recht anschaulich. Visualisierung in der Juristenausbildung, Köln 2007.

Sandkühler, Thomas/Bühl-Gramer, Charlotte/John, Anke/Schwabe, Astrid/Bernhardt, Markus (Hrsg.), Geschichtsunterricht im 21. Jahrhundert. Eine geschichtsdidaktische Standortbestimmung, Bonn 2018

Schiemann, Gottfried, Das Studium der Rechtsgeschichte in einer anwaltsorientierten Juristenausbildung, in: Hengstl/Sick (Hrsg.), Recht gestern und heute. Festschrift zum 85. Geburtstag von Richard Haase, Wiesbaden 2006, S. 193-201.

Schild, Wolfgang, Folter, Pranger, Scheiterhaufen. Rechtsprechung im Mittelalter, München 2010.

Schmidt-Wiegand, Ruth (Hrsg.), Deutsche Rechtsregeln und Rechtssprichwörter, München 1996.

Schmoeckel, Mathias/Stolte, Stefan (Hrsg.), Examinatorium Rechtsgeschichte, München 2008.

Schröder, Rainer, Lust auf Rechtsgeschichte? Vorwort zu seinem Alpmann-Schmidt-Skript Rechtsgeschichte, 9. Auflage, Münster 2013; Nachdruck in: Rechtslehre. Jahrbuch der Rechtsdidaktik 2013/2014, S. 97-101.

Stolleis, Michael, Zur kritischen Funktion der Rechtsgeschichte, in: Hof /v. Olenhusen (Hrsg.), Rechtsgestaltung - Rechtskritik - Konkurrenz von Rechtsordnungen .... Neue Akzente für die Juristenausbildung, Baden-Baden 2012, S. 212-219.

ders., Stärkung der Grundlagenfächer, in: JZ 2013, S. 712-714.

Wissenschaftsrat, Perspektiven der Rechtswissenschaft in Deutschland. Situation, Analysen, Empfehlungen, Hamburg 2012, Drs. 2558-12, online unter: https://www.wissenschaftsrat.de/download/archiv/2 558-12.pdf (16.07.2019).

Zwickel, Martin, Rechtsvergleichung und juristisches Lernen. Zu Parallelen zwischen Rechtsvergleichung und juristischer Methodenkompetenz (Fallbearbeitung und wissenschaftliches Arbeiten), in: Griebel (Hrsg.), Vom juristische Lernen, Baden-Baden 2018, S. 134-157. 